\title{
ANALGESIC EFFICACY OF COMBINATION OF BILATERAL SUPERFICIAL CERVICAL PLEXUS BLOCK AND STANDARD ANALGESIA WITH STANDARD ANALGESIA ALONE IN PATIENTS UNDERGOING THYROID CANCER SURGERY. A RANDOMISED-CONTROLLED TRIAL
}

\author{
Hammad N. Najeeb, Ahsan W. Khan, Athar M. Siddiqui \\ Department of Anesthesia, Shaukat Khanum Memorial Cancer Hospital and Research Centre, Lahore, Pakistan \\ Received: 22 January 2019 / Accepted: 14 June 2019
}

\begin{abstract}
Purpose: This study aimed to determine analgesic efficacy of a combination of bilateral superficial cervical plexus block (BLSCPB) and standard analgesia with standard analgesia alone in patients undergoing thyroid cancer surgery under general anaesthesia.

Materials and Methods: It was a randomised-controlled trial conducted at the Anaesthesia Department of the Shaukat Khanum Memorial Cancer Hospital, Lahore. A sample size of 60 patients (30 patients in each group) was calculated with $95 \%$ confidence interval and power of $80 \%$.

Results: The mean pain-free duration for the Group A (BLSCPB group) was 72 min (SD 30.1) compared to 53 min (standard deviation 30.3) for the Group B. This shows a significant reduction in pain-free duration in Group A. Average NRS pain score in the Group A at q 0, 30, 60, 90 and 120 min was (0.83, 1.83, 0.23, 1.76 and 1.49), whereas the average pain score on NRS in the Group B at the same time interval mentioned above for Group A was $(2.1,2.73$, $4.53,2.44$ and 1.85)
\end{abstract}

Conclusion: Administration of BLSCPB resulted in superior analgesia after thyroid cancer surgery.

Key words: Analgesia, cervical plexus block, post-operative pain, regional anaesthesia, thyroidectomy

\section{Introduction}

Common complications after thyroidectomy are infection, vocal cord paralysis, bleeding and post-operative pain. Recent advancements have minimised most of these surgical complications, but post-operative pain still remains as a significant problem, specifically in the day care settings. ${ }^{[1]}$ These patients usually complain of moderate intensity short duration pain. ${ }^{[2]} \mathrm{Up}$ to $90 \%$ patient receive opioids for its treatment on the $1^{\text {st }}$ post-operative day. ${ }^{[3]}$ There is high risk of nausea and vomiting after thyroid surgery, ${ }^{[4]}$ and uncontrolled pain can further exacerbate this problem. Opioids are the gold standard for post-operative pain. Due to their undesirable side effects such as nausea and vomiting, sedation and respiratory

Correspondence: Hammad N. Najeeb, Department of Anesthesia, Shaukat Khanum Memorial Cancer Hospital and Research Centre,

Lahore, Pakistan. Email: hammadnajeeb@gmail.com depression, anaesthesia practice has evolved from 'opioid based' to 'opioid sparing' or more recently into 'opioid free anaesthesia.' This requires non-opioid analgesics and use of regional analgesia techniques. Acetaminophen is a non-opioid analgesic, which does not cause postoperative nausea and vomiting, sedation or respiratory depression. It is a drug of choice for the management of post-operative pain, especially after thyroidectomy ${ }^{[5]}$ As part of multimodal analgesia regional blocks are gaining popularity in the management of post-operative pain after thyroidectomy.

A study of 162 patients who underwent thyroidectomy and received bilateral superficial cervical plexus block (BLSCPB), pain score was much lower in the initial hours post-surgery that eventually leads to a shortened hospital stay. Meantime duration to keep patient pain free in Group A (general anaesthesia [GA] + standard analgesia 
along with isotonic saline for BLSCPB) was $82.1 \pm 61 \mathrm{~min}$ versus in Group B (GA + standard analgesia with $0.5 \%$ bupivacaine for BLSCPB) was $360.8 \pm 213.75 \mathrm{~min}^{[6]}$ However, another similar study on 111 patients who were infiltrated with BLSCPB either before commencement or the end of surgery as a regional block with $0.75 \%$ ropivacaine concluded that there was no improvement of post-operative pain score. ${ }^{[7]}$ Alternatively, BLSCPB used in-patient undergoing carotid endarterectomy had improved analgesic efficacy as compared to combined (superficial and deep cervical plexus block) technique. ${ }^{[8-9]}$ Hence, information regarding the efficacy of BLSCBPs in post-operative analgesia for thyroid surgery has been controversial, with some studies ${ }^{[10]}$ proving BLSCBP to be effective while other studies ${ }^{[11]}$ finding no effect with the same.

Standard intravenous (IV) analgesia has not been established in literature; however, multimodal regimen currently used in our setup is comparable with other researches. These include a combination of opioids, nonsteroidal anti-inflammatory drugs and acetaminophen. For this study, we defined standard analgesia as 'IV morphine $0.1 \mathrm{mg} / \mathrm{kg}+15 \mathrm{mg} / \mathrm{kg}$ acetaminophen.' Since there are conflicting reports regarding the efficacy of BLSCPB, we used standard analgesia in both groups. In this way, we did not deprive our patients the current standard of care at our institute.

The aim of this study was to compare the analgesic efficacy in terms of duration of pain-free period after the administration of BLSCPB and standard analgesia with standard analgesia alone. The study outcome can help us to develop better analgesic modality for thyroidectomy patients. If proven to be efficacious, further studies may use cervical plexus block as a stand-alone technique or in combination with reduced number or dose of analgesics that we currently use as part of our standard regimen.

\section{Objectives}

The objective of the study is to compare the mean duration of patient's pain-free time in Group A (standard analgesia with BLSCPB) versus in Group B (Standard analgesia without BLSCPB) in patients undergoing thyroid cancer surgeries.

\section{Operational definitions}

Outcome variable was the patient's pain-free time. After the procedure, patients were shifted to post-anaesthesia care unit (PACU) where they were evaluated for post-operative pain using numeric pain rating score (10: The worst pain imaginable and 0: Pain free) at $\mathrm{q} 0$, $30,60,90$ and $120 \mathrm{~min}$. When numeric rating score was $\geq 4 / 10$, pain was labeled and duration of pain-free time was recorded.

\section{Bilateral superficial cervical plexus blocks}

The cervical plexus is made up of the ventral rami of the first four spinal nerves, which further distribute in superficial or deep motor branches. The block was performed using $20 \mathrm{ml}$ of $0.25 \%$ bupivacaine on each side of the neck along the dorsal edge of the sternocleidomastoid muscle.

\section{Standard analgesia}

Standard analgesic regimen used in this study was intravenous $(0.1 \mathrm{mg} / \mathrm{kg}$ morphine and $15 \mathrm{mg} / \mathrm{kg}$ acetaminophen).

\section{Hypothesis}

There is a difference in the meantime duration to keep patient pain free in standard analgesia with BLSCPB versus in patients with standard analgesics without BLSCPB in-patient undergoing thyroid cancer surgeries under GA.

\section{Materials and Methods}

\section{Study design}

This was a prospective, observer-blinded randomisedcontrolled trial.

\section{Setting}

This study was conducted at the Department of Anaesthesia and Pain Medicine, Shaukat Khanum Memorial Cancer Hospital and Research Center, Lahore.

\section{Duration of study}

6 months. (From September 2017 to March 2018). 


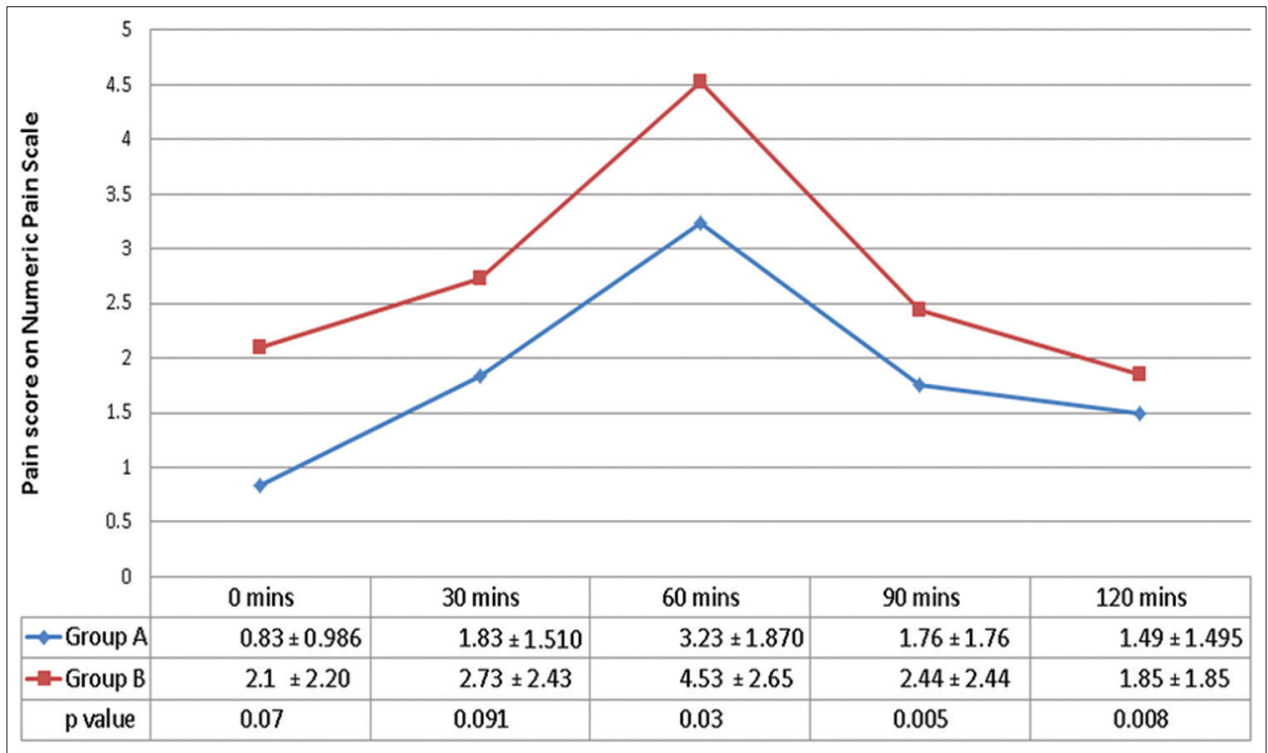

Graph 1: Mean pain score versus time intervals

\section{Table 1: Demographics characters and duration of surgery}

\begin{tabular}{l|c|c}
\hline Patient demographics & $\begin{array}{c}\text { BLSCPB } \\
(\boldsymbol{n}=\mathbf{3 0})\end{array}$ & $\begin{array}{c}\text { Control } \\
(\boldsymbol{n}=\mathbf{3 0})\end{array}$ \\
\hline Age (years) & $37.33 \pm 10$ & $37.01 \pm 8$ \\
\hline Weight (kg) & $66 \pm 9$ & $69 \pm 6$ \\
\hline Male: Female $(n)$ & $3: 7$ & $2: 8$ \\
\hline Duration of surgery (min) & $130 \pm 41$ & $128 \pm 27$ \\
\hline
\end{tabular}

Table 2: Mean pain-free duration with $P$ value

\begin{tabular}{l|c|c|c|c}
\hline Age (year) & Groups & \multicolumn{2}{|c|}{$\begin{array}{c}\text { Pain-free } \\
\text { duration }\end{array}$} & \multirow{2}{*}{ P value } \\
\cline { 3 - 4 } & & $\boldsymbol{n}$ & Mean \pm SD & \\
\hline$\leq 30$ & Group A & 10 & $70 \pm 28.5$ & \multirow{2}{*}{0.003} \\
\cline { 2 - 4 } & Group B & 10 & $49 \pm 23.6$ & \\
\hline$\geq 30$ & Group A & 20 & $74 \pm 31.7$ & \multirow{2}{*}{0.048} \\
\cline { 2 - 4 } & Group B & 20 & $57 \pm 37.0$ & \\
\hline
\end{tabular}

SD: Standard deviation

\section{Sample size}

Sample size of 60 patients (30 patients in each group) calculated based on the pioneer study ${ }^{[10]}$ with $95 \%$ confidence interval, $80 \%$ power of the test and expected meantime to keep the patient pain free. A difference pain-free duration of $130 \mathrm{~min}$ (standard deviation [SD]
$45 \pm 10$ ) was observed in the reference study between the 2 groups.

\section{Sampling technique}

Non-probability Consecutive sampling.

\section{Inclusion criteria}

1. American Society of Anesthesiologists (ASA) I and II

2. Patients 20-60 years of age of either gender undergoing thyroidectomy for thyroid cancer.

\section{Exclusion criteria}

1. Patients refusal for intervention

2. Patient allergic to bupivacaine and opioids

3. Renal abnormality with (creatinine value $>2.0$ )

4. Patients with a history of smoking, chronic narcotic use or alcohol abuse.

\section{Data collection}

After getting the approval from Hospital Ethics Committee, patients who met the inclusion criteria were randomly allocated to groups using random number table available online. Group A was patients receiving BLSCPB, and Group B was patients not receiving BLSCPB. Both groups received standard analgesia. 
All patients were provided with patient information sheet, and written informed consent was obtained. The demographic information, ASA status, age and weight were noted. IV access was established in all patients, and vitals were checked before the induction. Patients were shifted to operating room, and ASA standard monitoring (electrocardiography, non-invasive blood pressure, heart rate and pulse oximetry) was applied. A standard anesthetic regimen was applied. Anaesthesia was induced with propofol $1-2 \mathrm{mg} / \mathrm{kg}$ and muscle relaxation with atracurium $0.5 \mathrm{mg} / \mathrm{kg}$. Airway was secured by endotracheal tube. All patients were ventilated with volume controlled ventilation with a mixture of $50 \%$ oxygen and 50\% air and 1.5-2.5\% sevoflurane.

Standard analgesia $(0.1 \mathrm{mg} / \mathrm{kg}$ morphine and $15 \mathrm{mg} / \mathrm{kg}$ acetaminophen for pain management) was given to the patients in both groups. Group A received standard analgesia and regional block (BLSCPB) with $20 \mathrm{ml}$ of $0.25 \%$ bupivacaine using 3 point injection technique, and the spread of local anaesthesia was visualised with the ultrasound machine before the commencement of surgery.

Group B received GA and standard analgesia without the regional block (BLSCPB). All patients were administered IV fluid consisting of Ringer's lactate solution for maintenance according to patient's weight.

After the surgery, patients were shifted to the PACU. They were evaluated for pain using numeric pain rating score, at $0,30,60,90$ and 20 min after surgery. If the patient's pain score was higher than four, a rescue analgesia of diclofenac $75 \mathrm{mg}$ was given. Pain-free period was recorded as per operational definition.

\section{Statistical analysis}

Statistical analysis was performed using SPSS version 22 for IBM (SPSS, Chicago, Illinois, USA). Quantitative variable was presented as mean \pm SD. Qualitative variables such as gender were presented as frequency and percentage. Both groups were compared by applying $t$-test. Assumptions for $t$-test (normality and equality of variance) were checked using graphical and statistical test. Post-stratification $t$-test was applied with $P<0.05$ as statistically significant.

\section{Results}

\section{Age distribution}

Table 1 demonstrates demographic characteristic and duration of surgery. $10(33.3 \%)$ patients in Group A were $\leq 30$ years and $20(66.6 \%)$ patients in Group B were more than 30 years of age. Similarly, $10(33.3 \%)$ patients in Group A were more $\leq 30$ years of age and $20(66.6 \%)$ patients were more than 30 years of age in Group B. Mean age of the patients was 37.33 years in the Group A and 37.0 in Group B, the range being 18-60 years.

\section{Gender distribution}

Group A consisted of 21 female (60\%) and 9 (30\%) male patients. Group B consisted of $24(80 \%)$ females and $6(20 \%)$ males.

\section{Mean pain-free duration (primary outcome)}

The mean pain-free duration for the Group A was 72 min $( \pm 30.1)$ compared to a mean free duration of $53 \mathrm{~min}$ $( \pm 30.3)$ for Group B.

Patients whose age was $\leq 30$ years in Group A had a mean pain-free duration of $70 \mathrm{~min}( \pm 28.5)$ as compared to Group B where mean pain-free duration was $49 \mathrm{~min}$ ( \pm 23.6). Similarly, patients whose age was more than 30 years in Group A had a mean pain-free duration of $74 \mathrm{~min}( \pm 31.7)$ as compared to Group B where mean pain-free duration was $57 \mathrm{~min}( \pm 37)$. This shows a significant reduction in mean pain-free duration in Group A population [Table 2].

\section{Mean pain score}

Average NRS pain score in the Group A at q 0, 30, 60, 90 and 120 minutes was $(0.83 \pm 0.986,1.83 \pm 1.510$, $3.23 \pm 1.870,1.76 \pm 1.76$ and $1.49 \pm 1.495)$, whereas the average NRS pain score in the Group B at q 0, 30, 60, 90 and 120 minutes was $(2.1 \pm 2.20,2.73 \pm 2.43,4.53 \pm$ $2.65,2.44 \pm 2.44$ and $1.85 \pm 1.85$ ) [Graph 1].

\section{Discussion}

Literature regarding the analgesic efficacy of bilateral BLSCPB for post-operative analgesia after thyroidectomy remains inclusive. While some studies ${ }^{[6,11]}$ have found no 
benefit for post-operative pain control with BLSCPSB, others advocated BLSCPB as a promising and effective analgesic modality in patients undergoing thyroidectomy. ${ }^{[7]}$ This study can be differentiated from other similar studies as this study included only thyroid carcinoma patients undergoing thyroidectomy.

The goal of this study was to compare the analgesic efficacy by calculating the mean pain-free duration of the BLSCPB group versus the control group. BLSCPB was performed immediately after the induction of GA using the three-point landmark technique and then visualising the spread of bupivacaine with an ultrasound machine. We used $0.25 \%$ bupivacaine with GA versus standard analgesia alone being used at our setup for post-operative pain management. There was significant difference in pain scores at all time intervals in our study. These results suggest that there is a considerable decrease in pain scores in the first $2 \mathrm{~h}$ after the surgery in the BLSCPB patient as compared to the standard analgesia alone group. It was also observed that fewer patients in Group A required rescue analgesic dose of $75 \mathrm{mg}$ diclofenac as compared to the control group.

The choice of drug to be used in the cervical block is debatable, and various combinations are recommended in different studies. In the study by Andieu et al., clonidine was used as an adjuvant with bupivacaine for regional anaesthesia, this lead to a significant decrease in perioperative analgesic requirement in patients of BLSCPB group. Clonidine enhances pain relief after peripheral nerve block, acting through a central action due to systemic absorption and a direct action on local nerve fibres. ${ }^{[12]}$ We did not use clonidine as it is not available in our country.

In another study by Eti et al., ${ }^{[11]}$ patients did not require additional analgesia for initial hours when BLSCPB was performed at the end of thyroidectomy; furthermore, $34 \%$ did not require opiates at all in the first $24 \mathrm{~h}$ after surgery.

Our results are comparable to available literature. This study shows that BLSCPB is an effective method of reducing post-operative pain after thyroidectomy. Postoperative pain was well controlled during the stay in PACU. Additional analgesia paracetamol was prescribed as the patient was discharged from PACU. This way the use of opioid was minimised in the post-operative period and hence the associated side effects such as nausea and vomiting.

Meantime duration of pain-free period was longer in the BLSCPB patients, however it was shorter than what was expected. There could be two possible explanations. First, few patients had a previous thyroid surgery which could have distorted the anatomical planes of thyroid. This could alter the spread of the bupivacaine infiltrated in the region. Hence, these patients had a lesser pain satisfaction score, some of our patient population consisted of non-Urdu/ English speaking group. We required a translator (usually an attendant) to translate the pain score. Language barrier and patient apprehension could have been a factor in undermining the pain score. Hence, this is considered as another limitation of the study.

The pain scores in Group B confirm that some patients experience moderate/severe pain (mean pain score $>4$ at $60 \mathrm{~min}$ ) after surgery but for a short duration. Acetaminophen alone was insufficient, and rescue analgesic dose of diclofenac $75 \mathrm{mg}$ was added in these patients. Hence, regional anaesthesia is an appropriate component of multimodal analgesia in this setting.

\section{Conclusion}

BLSCPB infiltrated after the induction of GA is effective method of reducing post-operative pain after thyroid cancer surgery. It also significantly decreases the severity of pain in the first $2 \mathrm{~h}$ after surgery. Further studies are required with larger sample size to establish the role of cervical plexus block as an effective modality for preventing post-operative pain after thyroid cancer surgeries.

\section{Conflict of Interest}

The authors declare that they have no conflict of interest.

\section{References}

1. Santosh BS, Mehandale SG. Does dexmedetomidine improve analgesia of superficial cervical plexus block for thyroid surgery? Indian J Anaesth 2016;60:34-8.

2. Motamed C, Merle JC, Yakhou L, et al. Intraoperative i.v. Morphine reduces pain scores and length of stay in the post anaesthetic care unit after thyroidectomy. Br J Anaesth 
2004;93:306-7.

3. Gozal Y, Shapira SC, Gozal D, et al. Bupivacaine wound infiltration in thyroid surgery reduces postoperative pain and opioid demand. Acta Anaesthesiol Scand 1994;38:813-5.

4. Sonner JM, Hynson JM, Clark O, et al. Nausea and vomiting following thyroid and parathyroid surgery. J Clin Anesth 1997;9:398-402.

5. Soltani G, Molkizadeh A, Amini S. Effect of intravenous acetaminophen (paracetamol) on hemodynamic parameters following endotracheal tube intubation and postoperative pain in caesarian section surgeries. Anesth Pain Med 2015;5:e30062.

6. Shih ML, Duh QY, Hsieh CB, et al. Bilateral superficial cervical plexus block combined with general anesthesia administered in thyroid operations. World J Surg 2010;34:2338-43.

7. Herbland A, Cantini O, Reynier P, et al. The bilateral superficial cervical plexus block with $0.75 \%$ ropivacaine administered before or after surgery does not prevent postoperative pain after total thyroidectomy. Reg Anesth Pain Med 2006;31:34-9.

8. Ivanec Z, Mazul-Sunkol B, Lovricević I, et al. Superficial versus combined (deep and superficial) cervical plexus block for carotid endarterectomy. Acta Clin Croat
2008;47:81-6.

9. Andrieu G, Amrouni H, Robin E, et al. Analgesic efficacy of bilateral superficial cervical plexus block administered before thyroid surgery under general anaesthesia. Br J Anaesth 2007;99:561-6.

10. Suh YJ, Kim YS, In JH, et al. Comparison of analgesic efficacy between bilateral superficial and combined (superficial and deep) cervical plexus block administered before thyroid surgery. Eur J Anaesthesiol 2009;26:1043-7.

11. Eti Z, Irmak P, Gulluoglu BM, et al. Does bilateral superficial cervical plexus block decrease analgesic requirement after thyroid surgery? Anesth Analg 2006;102:1174-6.

12. Hutschala D, Mascher H, Schmetterer L, et al. Clonidine added to bupivacaine enhances and prolongs analgesia after brachial plexus block via a local mechanism in healthy volunteers. Eur J Anaesthesiol 2004;21:198-204.

\section{Authorship Contributions}

Concept and design: HNN, AWK, AMS; Data Collection and interpretation: HNN; Literature review and writing: HNN, AMS; Manuscript approval HNN, AWK, AMS 